\title{
Revisión de experiencias de gamificación en la enseñanza de lenguas extranjeras
}

Beatriz Chaves Yuste - Universidad Antonio de Nebrija

$0000-0002-4442-9138$

Recepción: 22.07.2019 | Aceptado: 21.11.2019

Correspondencia a través de ORCID: Beatriz Chaves Yuste

0000-0002-4442-9138

Citar: Chaves Yuste, B. (2019). Revisión de experiencias de gamificación en la enseñanza de lenguas extranjeras. ReiDoCrea, 8, 422-430.

Resumen: Antecedentes. Tras el continuado uso del método comunicativo en la enseñanza de lenguas extranjeras durante las últimas décadas, se han desarrollado un elenco de herramientas atractivas y motivadoras que pueden aplicarse fácilmente en el marco de la educación. Las TIC han promovido el uso de aplicaciones móviles y de videojuegos como instrumentos educativos en un entorno de gamificación. La gamificación ayuda a los estudiantes de lenguas extranjeras de todas las edades y niveles a experimentar un aprendizaje que fomenta su participación y responsabilidad como agentes de su propio aprendizaje. Objetivo/ hipótesis. Realizar un análisis exhaustivo de la literatura científica sobre el tema. Método. Se utilizan las bases de datos Dialnet, Google Scholar, Science Direct, Redalyc y ERIC desde el año 2015 hasta la actualidad. Resultados. Se hallan 30 publicaciones sobre gamificación en lengua extranjera siendo 14 españoles en los últimos cinco años. Conclusión. La gamificación se ha consolidado como un nuevo método de aprendizaje donde se diseñan entornos motivadores de aprendizaje significativo, se exponen experiencias gamificadas digitales y analógicas y se fomenta el aprendizaje cooperativo y colaborativo para mejorar la competencia lingüística del alumnado.

Palabras clave: Innovación Pedagógica | Motivación

\section{Revision of Gamification Experiences for Foreign Languages Teaching}

Abstract: Background. After the continuous implementation of the communicative approach when teaching foreign languages during the last decades, the current society offers a wide range of attractive and motivating tools that can be easily used in education. The use of ICT, mobile apps and videogames comprise both a wide educational and funny experience. Gamification helps foreign languages students (no matter their age or level), experiment a more complex learning in which they are active and responsible participants of their own learning. Objectives/hypotheses. Perform an exhaustive analysis of the scientific literature on the subject. Method. The Dialnet, Google Scholar, Science Direct, Redalyc and ERIC databases from 2015 to the present are used. Results. There are 30 publications on gamification in a foreign language with 14 Spaniards in the last five years. Conclusions. The consolidation of gamification as a new learning method. Thanks to gamification, meaningful and motivating learning environments take place, digital and analogical game experiences are displayed and cooperative and collaborative learning is promoted while improving students' communicative competence.

Keywords: Teaching Method Innovations | Motivation

\section{Introducción}

El juego es una actividad significativa y común en todas las culturas (Huizinga, 1949). Como defiende Mora (2013), la actividad lúdica ayuda a despertar la curiosidad, dirigir la atención hacia un punto determinado y fortalecer la memoria, lo cual, aplicado a un proceso de aprendizaje, por ejemplo, de una lengua, facilita la asimilación y procesamiento de léxico y estructuras sintácticas y morfológicas. En el aula de lenguas extranjeras el juego es una herramienta muy socorrida: como señala Foncubierta (2014), los juegos añaden diversión, mejoran la atención, ayudan a modular estados anímicos, a luchar contra la desidia y el aburrimiento en las aulas y proveen de descansos cuando se detecta agotamiento en el aprendizaje. 
La gamificación ha sido una herramienta de aprendizaje muy explotada en el mundo empresarial durante décadas. Tal ha sido su éxito, que su práctica se ha extrapolado a otros contextos, como, por ejemplo, el ámbito de la Enseñanza, fundamentalmente, en el contexto del aprendizaje-adquisición de lenguas extranjeras. La gamificación consiste en "the use of game design elements in non-game contexts" (Deterding, Dixon, Khaled, y Nacke, 2011, 10), en "el uso de elementos y técnicas de diseño de juegos en contextos ajenos al juego" (Werbach y Hunter, 2012, 26). Estos elementos y técnicas propias de los juegos se pueden explotar para promover la docencia de lenguas extranjeras de una manera gratificante y motivadora, muy útil en contextos donde el aprendiente no encuentra suficiente motivación intrínseca para aprender. Harmer y Lee (2011) afirman que la gamificación puede ser utilizada como método de intervención cognitiva, emocional y social, lo cual, aplicado al proceso de aprendizaje de una lengua extranjera supone el uso de técnicas del juego que hacen posible que la experiencia educativa sea más motivadora para el alumnado. Werbach (2012) establece seis pasos necesarios para diseñar un marco óptimo gamificado:

1. Definición de objetivos.

2. Delineación de las actitudes que se esperan de los aprendientes.

3. Descripción de los jugadores.

4. Diseño de la secuenciación de los ciclos de actividades (ciclos de implicación el estudiante se siente motivado a seguir su aprendizaje tras haber pasado una prueba y haber recibido puntos o insignias- o ciclos de progresión -todos los pasos que realiza el aprendiente son necesarios para poder alcanzar el objetivo final del juego. Dicho con otras palabras, el cumplimiento de varios objetivos a corto plazo hace que se alcancen objetivos a medio plazo y, por ende, tras cumplir con varios objetivos a medio plazo, se alcanza el objetivo a largo plazo final establecido en el juego.

5. Elaboración de herramientas de aprendizaje amenas para diferentes tipos de alumnado.

6. Implantación de herramientas apropiadas teniendo en cuenta la realidad educativa en la que la práctica gamificada tiene lugar.

Además, Werbach y Hunter (2012) señalan una serie de elementos derivados del juego: dinámicas, mecánicas y componentes de gamificación, generalmente representados bajo una forma piramidal donde se agrupan desde los elementos más abstractos a los más concretos. Las dinámicas son elementos que están presentes en casi todos los juegos y suponen el nivel más alto de abstracción. Entre las dinámicas más populares encontramos restricciones para los jugadores en la realización de ciertas actividades, las emociones, como la curiosidad, la satisfacción o la decepción al conseguir o no ciertos objetivos propuestos, la narrativa, que proporciona el marco estructural para desarrollar las diferentes pruebas, y la progresión de los participantes, que ayuda a visualizar cómo avanzan en las diferentes actividades y las relaciones, que muestran la interacción de los jugadores, su cohesión como grupo y la competitividad entre ellos. Por mecánicas se entienden elementos más específicos que implican acciones detalladas y dirigen a los jugadores hacia la dirección deseada para cumplir con los objetivos establecidos. Se pueden introducir varias mecánicas dentro de la misma dinámica (e.g. retroalimentación y recompensas para expresar progresión). Así pues, se consideran mecánicas de gamificación los retos, la competición y la cooperación, la retroalimentación, la adquisición de recursos o recompensas, las transacciones y los turnos. Por otro lado, los componentes son elementos necesarios para el funcionamiento de las mecánicas del juego. Se encuentran en el nivel más bajo de abstracción, pues son acciones muy concretas, cuyos componentes pueden ser logros, 
avatares, insignias, peleas heroicas, colecciones, combates, desbloqueo de contenido, regalos, tablas de clasificación, niveles, puntos, cruzadas, gráficos sociales, equipos y bienes virtuales.

Bartle (1996), por su parte, establece una taxonomía de los tipos de jugadores que se pueden encontrar en los potenciales usuarios de la experiencia gamificada:

a) Triunfadores: aquellos jugadores que desean reconocimiento por su labor y cuyo objetivo es ganar el juego.

b) Socializadores: jugadores que disfrutan del tiempo compartido con sus compañeros pues disfrutan siendo parte de una comunidad. La experiencia social que el juego conlleva es más importante que alcanzar los objetivos fijados.

c) Exploradores: les gusta adquirir nuevos conocimientos, interactuar con el mundo del juego y aprender con la actividad.

d) Asesinos: únicamente juegan para demostrar su superioridad por encima del resto de compañeros.

Esta taxonomía se suele representar con la siguiente figura:

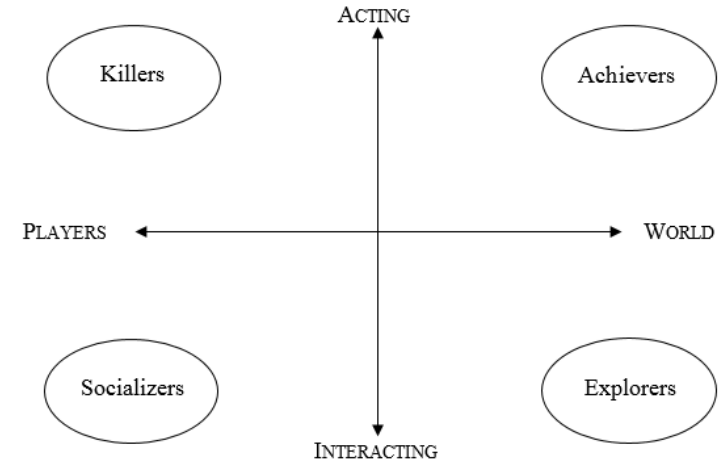

Figura 1. Bartle's taxonomy of player types (http://mud.co.uk/richard/hcds.htm)

El eje horizontal representa la preferencia de participar junto a otros jugadores e interactuar con el mundo. El eje vertical refleja una preferencia para interactuar con algo. Así pues, los triunfadores, por ejemplo, prefieren actuar en el mundo mientras que los socializadores desean estar con otros jugadores.

Como señala Barros (2016), algunas de las teorías que podrían operar la gamificación serían la motivación intrínseca de Pink (2010) (basada en la teoría de la autodeterminación de Ryan y Deci, 1997) y la teoría del flujo de Csíkszentmihályi (1990). Pink (2010) afirma que una persona estará más motivada cuando aquella actividad que realiza le produce satisfacción. La teoría del flujo, por su parte, defiende que el grado de involucración de una persona en una actividad depende de su grado de concentración.

Llegados a esto punto, tendríamos que preguntarnos: ¿en qué se diferencia la gamificación del aprendizaje basado en juegos (en adelante ABJ)? El ABJ consiste en el uso de juegos con objetivos educativos, utilizándolos como herramientas que apoyan los procesos de aprendizaje de forma significativa. Así pues, los contenidos se adaptan 
a la historia o escena del juego en sí, que tiene unas reglas y objetivos definidos. Este tipo de metodologías viene acompañado de otras tales como el Aprendizaje Basado en Proyectos (ABP) o en tareas (ABT). Sin embargo, a diferencia de la gamificación, los alumnos no consiguen puntos o insignias como parte de su evolución en la materia en sí durante la sesión o la unidad didáctica tratada, sino que ganan o pierden el juego que está incluido en dicha sesión o unidad didáctica como herramienta de apoyo en el aprendizaje, asimilación o evaluación de los conocimientos impartidos. Con ambas metodologías, gamificación y ABJ, los estudiantes están inmersos en un proceso de aprendizaje activo y autodirigido (learning by doing). Los alumnos se sienten altamente motivados y se promueve su autonomía y razonamiento en un aprendizaje activo, donde ellos reciben una retroalimentación o feedback instantáneo y donde además se potencia su creatividad, imaginación y habilidad social. Más aún, facilitan el desarrollo de la competencia digital ya que hay una infinidad de posibilidades para introducir juegos online, videojuegos o aplicaciones lúdicas que ayudan, a la par de asimilar, afianzar o evaluar conocimientos, a mejorar el uso de las nuevas tecnologías en un entorno seguro y diseñado para el aprendizaje.

\section{Objetivos o hipótesis}

Con este trabajo se pretende hacer una revisión bibliográfica sobre el uso de la gamificación en la enseñanza de lenguas extranjeras desde el 2015 hasta la actualidad con el fin de analizar los resultados obtenidos durante el periodo referido.

\section{Métodos}

El método utilizado en el presente trabajo se enfoca en el análisis de los estudios empíricos basados en experiencias educativas gamificadas para la enseñanza de lenguas extranjeras durante los últimos cinco años (desde el año 2015 hasta la actualidad), encontrados en las bases de datos Dialnet, Google Scholar, Science Direct, Redalyc y ERIC. Los descriptores usados en la búsqueda de las bases de datos son: gamificación y enseñanza lenguas. Para acotar la búsqueda se utilizaron los operadores booleanos AND / Y. Tras analizar con detalle toda la literatura volcada a través de los buscadores mencionados anteriormente, solamente treinta investigaciones (veinticinco escritas en español y cinco en inglés) incluían estudios que versan exclusivamente sobre gamificación llevadas a cabo en el aula de lengua extranjera independientemente de la lengua objeto de estudio, nivel de competencia lingüística, nivel educativo, edades de los participantes de la muestra y país donde el estudio tuvo lugar.

\section{Resultados}

En la tabla 1, se expone un resumen de los resultados obtenidos sobre estudios empíricos basados en el uso de la gamificación para impartir lenguas extranjeras durante los últimos cinco años. La tabla recoge los autores y títulos de la investigación, países donde se realizó el estudio, los objetivos de la investigación, los colectivos a los que se aplicó la metodología y las conclusiones extraídas de dichos estudios.

\begin{tabular}{|c|c|c|c|c|}
\hline AUTOR/ES (AÑO) & PAIIS & OBJETIVO & MUESTRA & CONCLUSIONES \\
\hline $\begin{array}{l}\text { BÁRCENA, E. Y } \\
\text { SANFILIPPO, M. } \\
\text { (2015). }\end{array}$ & España & $\begin{array}{l}\text { Explorar el valor de recursos } \\
\text { didácticos de tipo lúdico en el } \\
\text { aprendizaje de lenguas en un contexto } \\
\text { digital en formato audiovisual. }\end{array}$ & $\begin{array}{l}273 \text { estudiantes universitarios (133 } \\
\text { estudian inglés, } 77 \text { alemán, } 36 \\
\text { francés y } 27 \text { italiano). }\end{array}$ & $\begin{array}{l}\text { Actitud positiva y colaborativa de los } \\
\text { estudiantes. Conveniencia de } \\
\text { incorporar información audiovisual } \\
\text { de manera lúdica en plataformas } \\
\text { virtuales universitarias para } \\
\text { enseñanza de lenguas extranjeras. }\end{array}$ \\
\hline BATTLE, J. (2016). & Alemania & $\begin{array}{l}\text { Presentar una experiencia práctica } \\
\text { gamificada en la clase de ELE y } \\
\text { realizar valoración acerca de su } \\
\text { efectividad. }\end{array}$ & $\begin{array}{l}4 \text { estudiantes de Máster en } \\
\text { Comunicación y Mediación } \\
\text { Intercultural que estudian ELE }\end{array}$ & $\begin{array}{l}\text { Buena predisposición del alumnado } \\
\text { para trabajar con la práctica } \\
\text { gamificada. }\end{array}$ \\
\hline
\end{tabular}




\begin{tabular}{|c|c|c|c|c|}
\hline & & & $\begin{array}{l}\text { (Hochschule für Angewandte } \\
\text { Sprachen, Múnich). Nivel C1. }\end{array}$ & $\begin{array}{l}\text { La gamificación es una buena } \\
\text { herramienta pedagógica para } \\
\text { trabajar determinados contenidos. }\end{array}$ \\
\hline $\begin{array}{l}\text { BATLLE, J., } \\
\text { GONZÁLEZ, M. V. Y } \\
\text { PUJOLÀ, J. T. } \\
\text { (2018). }\end{array}$ & España & $\begin{array}{l}\text { Entender y mejorar el aprendizaje en } \\
\text { el aula de lenguas extranjeras. } \\
\text { Comprender cómo se plantea la } \\
\text { narrativa en propuestas didácticas } \\
\text { gamificadas. }\end{array}$ & $\begin{array}{l}16 \text { profesores de lenguas } \\
\text { extranjeras. }\end{array}$ & $\begin{array}{l}\text { Las secuencias didácticas } \\
\text { gamificadas que tienen narrativa y } \\
\text { no se centran exclusivamente en los } \\
\text { componentes apelan y aumentan el } \\
\text { divertimento y la motivación del } \\
\text { alumnado. }\end{array}$ \\
\hline $\begin{array}{l}\text { BATLLE, J. Y } \\
\text { SUÁREZ, M. M. } \\
\text { (2019). }\end{array}$ & España & $\begin{array}{l}\text { Analizar la interrelación de los PBL en } \\
\text { las unidades didácticas y su relación } \\
\text { con el desarrollo de habilidades } \\
\text { comunicativas (destrezas orales y } \\
\text { escritas) y el aprendizaje de } \\
\text { elementos del sistema de la lengua } \\
\text { (vocabulario, gramática y fonética). }\end{array}$ & $\begin{array}{l}21 \text { profesores de lenguas } \\
\text { extranjeras (EEOOII de Cataluña). }\end{array}$ & $\begin{array}{l}\text { Preferencia por la gamificación } \\
\text { superficial mediante PBL (17 de las } \\
21 \text { propuestas). } \\
\text { Mayor cantidad de actividades de } \\
\text { desarrollo de habilidades } \\
\text { comunicativas. } \\
\text { Preferencia por el uso combinado } \\
\text { de insignias y puntos. }\end{array}$ \\
\hline $\begin{array}{l}\text { CABARIQUE, L. I. } \\
\text { (2018). }\end{array}$ & Colombia & $\begin{array}{l}\text { Reconocer la influencia de los juegos } \\
\text { de programas tipo talk show } \\
\text { trabajados bajo elementos de la } \\
\text { gamificación en el desempeño de la } \\
\text { producción oral en inglés en adultos } \\
\text { de la fundación ColombiaCrece }\end{array}$ & $\begin{array}{l}6 \text { estudiantes adultos del } \\
\text { ColombiaCrece que estudian inglés. }\end{array}$ & $\begin{array}{l}\text { Los juegos trabajados con } \\
\text { elementos gamificados fueron } \\
\text { efectivos y tuvieron una influencia } \\
\text { positiva en el desempeño de la } \\
\text { producción oral de los estudiantes. } \\
\text { Mejoria entre los resultados del } \\
\text { examen inicial y final. Reducción de } \\
\text { niveles de ansiedad y presión. }\end{array}$ \\
\hline $\begin{array}{l}\text { CARRIÓN- } \\
\text { SALINAS, G. A. } \\
\text { (2017). }\end{array}$ & Ecuador & $\begin{array}{l}\text { Analizar las posibilidades de } \\
\text { gamificación como técnica de la } \\
\text { enseñanza y aprendizaje para ofrecer } \\
\text { una propuesta innovadora de } \\
\text { gamificación en educación primaria }\end{array}$ & $\begin{array}{l}46 \text { estudiantes de } 7^{\circ} \text { año de } \\
\text { educación básica ( } 11-12 \text { años) que } \\
\text { estudian inglés de la Unidad } \\
\text { Educativa de Milenio El Tambo } \\
\text { (Loja) divididos en dos grupos }\end{array}$ & $\begin{array}{l}\text { El } 65 \% \text { del alumnado se siente } \\
\text { motivado y participa más } \\
\text { activamente en las actividades } \\
\text { propuestas en el aula. }\end{array}$ \\
\hline $\begin{array}{l}\text { CORDERO, D. Y } \\
\text { NÚÑEZ, V. M. } \\
\text { (2018). }\end{array}$ & Costa Rica & $\begin{array}{l}\text { Desarrollar las competencias } \\
\text { lingüísticas en el área de Inglés } \\
\text { (habilidades escritas y orales) y } \\
\text { combinar el uso del idioma con el } \\
\text { entretenimiento y disfrute. }\end{array}$ & $\begin{array}{l}60 \text { estudiantes universitarios de la } \\
\text { Universidad Nacional que estudian } \\
\text { Inglés Integrado II (nivel A1). }\end{array}$ & $\begin{array}{l}\text { El uso de la gamificación } \\
\text { incrementa la motivación, } \\
\text { implicación y participación del } \\
\text { alumnado en su proceso de } \\
\text { aprendizaje. } \\
\text { Se genera un aprendizaje } \\
\text { competitivo y cooperativo. } \\
\text { Los alumnos son más responsables } \\
\text { de su propio aprendizaje. }\end{array}$ \\
\hline CRUAUD, C. (2017). & Noruega & $\begin{array}{l}\text { Producir aplicaciones digitales } \\
\text { innovadoras adaptadas a la necesidad } \\
\text { del profesorado y desarrollar nuevos } \\
\text { modelos de instrucción y aprendizaje. } \\
\text { Comprobar la eficacia del uso de la } \\
\text { gamificación en la enseñanza del } \\
\text { francés como lengua extranjera. }\end{array}$ & $\begin{array}{l}13 \text { alumnos de } 1^{0} \text { bachillerato ( } 16-17 \\
\text { años) que estudian francés. }\end{array}$ & $\begin{array}{l}\text { El uso de la gamificación } \\
\text { incrementa la implicación y } \\
\text { participación del alumnado en su } \\
\text { proceso de aprendizaje. }\end{array}$ \\
\hline $\begin{array}{l}\text { DALMASES, A. } \\
\text { (2017). }\end{array}$ & Filipinas & $\begin{array}{l}\text { Comprobar si la gamificación favorece } \\
\text { la consecución de objetivos de } \\
\text { aprendizaje establecidos teniendo en } \\
\text { cuenta las diferentes necesidades del } \\
\text { alumnado. }\end{array}$ & $\begin{array}{l}13 \text { alumnos filipinos con nivel } A 2 \\
\text { ( } 25-40 \text { años) que estudian ELE en el } \\
\text { Instituto Cervantes de Manila. }\end{array}$ & $\begin{array}{l}\text { La gamificación mejora el } \\
\text { comportamiento de los alumnos. } \\
\text { Mayor participación, implicación y } \\
\text { esfuerzo por parte del alumnado. }\end{array}$ \\
\hline $\begin{array}{l}\text { DÍAZ, J. J. Y CERDA } \\
\text { G. M. (2018). }\end{array}$ & Ecuador & $\begin{array}{l}\text { Estudio de las interferencias } \\
\text { sintáctico-morfológicas del español en } \\
\text { el proceso de la escritura en inglés. }\end{array}$ & $\begin{array}{l}50 \text { estudiantes universitarios que } \\
\text { estudian inglés (nivel A2) divididos } \\
\text { en dos grupos de } 25 \text { (grupo de } \\
\text { control y experimental). }\end{array}$ & $\begin{array}{l}\text { El uso de la gamificación contribuye } \\
\text { a una mejora metodológica. Los } \\
\text { estudiantes experimentan un } \\
\text { aprendizaje significativo e } \\
\text { interactúan, innovan y trabajan } \\
\text { colaborativamente en ambientes } \\
\text { motivados. }\end{array}$ \\
\hline $\begin{array}{l}\text { GALEANO, J. D. Y } \\
\text { RODRÍGUEZ L. E. } \\
\text { (2016). }\end{array}$ & Colombia & $\begin{array}{l}\text { Evaluar la incidencia de un videojuego } \\
\text { educativo apoyado en técnicas de } \\
\text { gamificación para adquirir vocabulario } \\
\text { y dominio de tiempos verbales en } \\
\text { inglés. }\end{array}$ & $\begin{array}{l}74 \text { estudiantes de } 5^{\circ} \text { grado ( } 10-11 \\
\text { años) que estudian inglés divididos } \\
\text { en dos grupos (control y } \\
\text { experimental) de la Institución } \\
\text { educativa Sikuani Ciudad Jardín } \\
\text { Nort (Suba). }\end{array}$ & $\begin{array}{l}\text { El uso de las técnicas de } \\
\text { gamificación en un videojuego } \\
\text { incide positivamente en el aumento } \\
\text { del dominio de vocabulario y } \\
\text { tiempos verbales en inglés. }\end{array}$ \\
\hline GARCÍA, F. (2017). & España & $\begin{array}{l}\text { Comprobar si el uso de la gamificación } \\
\text { en un aula de primaria aumenta la } \\
\text { motivación y la competencia lingüística } \\
\text { escrita en inglés. }\end{array}$ & $\begin{array}{l}\text { Dos grupos (control y experimental) } \\
\text { de estudiantes de } 5^{\circ} \text { curso de } \\
\text { educación primaria que estudian } \\
\text { inglés en un colegio público de la } \\
\text { Comunidad de Madrid. No } \\
\text { especifica el número de } \\
\text { participantes. }\end{array}$ & $\begin{array}{l}\text { El grupo experimental mostró un } \\
\text { incremento significativo frente a los } \\
\text { alumnos del grupo de control en el } \\
\text { grado de motivación hacia el estudio } \\
\text { de la lengua inglesa y en el nivel de } \\
\text { competencia lingüística en } \\
\text { comunicación escrita. }\end{array}$ \\
\hline $\begin{array}{l}\text { GARGALLO, P. } \\
\text { (2017). }\end{array}$ & España & $\begin{array}{l}\text { Diseñar y desarrollar una experiencia } \\
\text { educativa mediante el uso de tablets } \\
\text { para promover el uso del inglés en un } \\
\text { aula de Educación Infantil. }\end{array}$ & $\begin{array}{l}31 \text { alumnos de } 3^{\circ} \text { de educación } \\
\text { infantil ( } 5-6 \text { años) que estudian } \\
\text { inglés en un colegio de Burriana } \\
\text { (Castellón). }\end{array}$ & $\begin{array}{l}\text { Mejora significativa de la motivación } \\
\text { e interés por el aprendizaje de la } \\
\text { lengua inglesa. }\end{array}$ \\
\hline $\begin{array}{l}\text { GONZÁLEZ, D. } \\
\text { (2017). }\end{array}$ & España & $\begin{array}{l}\text { Diseñar e implantar una propuesta } \\
\text { didáctica en la que se aplique la } \\
\text { gamificación en el proceso de } \\
\text { enseñanza-aprendizaje del inglés. }\end{array}$ & $\begin{array}{l}24 \text { alumnos de } 2^{\circ} \text { educación } \\
\text { primaria que estudian inglés en el } \\
\text { colegio Sagrado Corazón HH } \\
\text { Salesianas de Burgos. }\end{array}$ & $\begin{array}{l}\text { Gran participación, interés y } \\
\text { motivación del alumnado. Muy } \\
\text { buenos resultados académicos. }\end{array}$ \\
\hline IBÁÑEZ, A. (2015). & España & $\begin{array}{l}\text { Comprobar si la aplicación Qstream u } \\
\text { otras con este tipo de formato (i.e. } \\
\text { quizzlet) puede responder a la } \\
\text { necesidad del alumnado de practicar } \\
\text { las competencias requeridas en } \\
\text { lengua inglesa de una manera lúdica, } \\
\text { promoviendo el trabajo colaborativo. }\end{array}$ & $\begin{array}{l}16 \text { estudiantes universitarios } \\
\text { (UNED) de la asignatura Lengua } \\
\text { Inglesa III para el Turismo y } 13 \text { de la } \\
\text { asignatura Inglés II para Turismo } \\
\text { (grupo experimental) y } 29 \text { alumnos } \\
\text { de las mismas asignaturas tomados } \\
\text { al azar (grupo de control) (nivel B1). }\end{array}$ & $\begin{array}{l}\text { Los alumnos del grupo experimental } \\
\text { tienen mejores resultados que los } \\
\text { alumnos del grupo de control. Los } \\
\text { estudiantes mejoran especialmente } \\
\text { los conocimientos gramaticales. } \\
\text { El uso de aplicaciones de } \\
\text { evaluación y autoevaluación con un } \\
\text { enfoque gamificador es motivador y }\end{array}$ \\
\hline
\end{tabular}




\begin{tabular}{|c|c|c|c|c|}
\hline & & $\begin{array}{l}\text { Promover el desarrollo de las } \\
\text { habilidades de producción escrita en } \\
\text { inglés y el desarrollo de la reflexión } \\
\text { sobre el propio proceso de } \\
\text { aprendizaje. }\end{array}$ & & $\begin{array}{l}\text { promueve el desarrollo y práctica de } \\
\text { destrezas lingüísticas. }\end{array}$ \\
\hline JIMÉNEZ, D. (2015). & $\begin{array}{l}\text { España, } \\
\text { EEUU, } \\
\text { Islandia y } \\
\text { Portugal. }\end{array}$ & $\begin{array}{l}\text { Comprobar si el empleo de estrategias } \\
\text { gamificadas tiene un efecto positivo } \\
\text { que motiva y mejora el rendimiento de } \\
\text { los estudiantes de ELE. }\end{array}$ & $\begin{array}{l}607 \text { estudiantes de España, Estados } \\
\text { Unidos, Islandia y Portugal que } \\
\text { estudian ELE. }\end{array}$ & $\begin{array}{l}\text { La gamificación mejora el } \\
\text { rendimiento y motivación del } \\
\text { alumnado en su proceso de } \\
\text { aprendizaje de ELE. }\end{array}$ \\
\hline $\begin{array}{l}\text { LAM, Y. W., HEW, K. } \\
\text { F. Y CHIU, K. F. } \\
\text { (2018). }\end{array}$ & Hong Kong & $\begin{array}{l}\text { Desarrollar un aprendizaje combinado } \\
\text { que mejore la escritura de textos } \\
\text { argumentativos en estudiantes de ESL } \\
\text { de educación secundaria. Determinar } \\
\text { si el uso de mecanismos de } \\
\text { gamificación en juegos digitales } \\
\text { incrementa la participación online del } \\
\text { alumnado y mejora su escritura de } \\
\text { textos argumentativos. }\end{array}$ & $\begin{array}{l}\text { Estudiantes de tres clases de } 4^{\circ} \\
\text { curso de educación secundaria (16- } \\
17 \text { años) que estudian inglés (un } \\
\text { grupo de control y dos experimental) } \\
\text { en un colegio femenino. No } \\
\text { especifica el número de } \\
\text { participantes. }\end{array}$ & $\begin{array}{l}\text { Conveniencia del aprendizaje } \\
\text { combinado para mejorar la escritura } \\
\text { de textos argumentativos. } \\
\text { El uso de la gamificación mejora el } \\
\text { aprendizaje del alumnado. }\end{array}$ \\
\hline $\begin{array}{l}\text { MARTÍN-MACHO, A. } \\
\text { Y FAYA, F. (2016). }\end{array}$ & España & $\begin{array}{l}\text { Ofrecer a los estudiantes una } \\
\text { oportunidad motivadora para afianzar } \\
\text { los contenidos lingüísticos ya } \\
\text { impartidos relacionados con aspectos } \\
\text { semánticos, sintácticos, pragmáticos y } \\
\text { fonéticos. } \\
\text { Dar a conocer las estrategias más } \\
\text { simples del juego cooperativo. }\end{array}$ & $\begin{array}{l}\text { Estudiantes de la asignatura Lengua } \\
\text { Inglesa y su Didáctica I del grado de } \\
\text { Maestro en Educación Infantil (nivel } \\
\text { B1). No especifica el número de } \\
\text { participantes. }\end{array}$ & $\begin{array}{l}\text { Incremento significativo de la } \\
\text { motivación del alumnado. } \\
\text { Interdependencia positiva e } \\
\text { interacción estimuladora del } \\
\text { aprendizaje cooperativo. }\end{array}$ \\
\hline $\begin{array}{l}\text { MONGROVEJO, A. } \\
\text { B. Y MAMANI, G. . Y } \\
\text { TIPO, M. L. (2019). }\end{array}$ & Perú & $\begin{array}{l}\text { Evaluar el dominio de la lengua } \\
\text { inglesa en expresión oral, la } \\
\text { comprensión auditiva, la expresión } \\
\text { escrita y el deletreo de vocabulario } \\
\text { sobre indumentaria, transporte, } \\
\text { profesiones, familia, partes del cuerpo } \\
\text { humano, deportes, nacionalidades, } \\
\text { objetos personales afines a la edad y } \\
\text { verbos de actividades cotidianas. }\end{array}$ & $\begin{array}{l}36 \text { alumnos hispanohablantes de } 4^{\circ} \\
\text { curso de educación secundaria del } \\
\text { IE Daniel Goleman (Juliaca) y } 20 \\
\text { alumnos del IE Jens Knudsen } \\
\text { (Arequipa) que estudian inglés (nivel } \\
\text { A1). }\end{array}$ & $\begin{array}{l}\text { Mejora significativa del aprendizaje } \\
\text { del vocabulario inglés en } \\
\text { estudiantes de habla hispana con } \\
\text { técnica altamente motivadora. } \\
\text { Diferencia de aprendizaje } \\
\text { significativa (en el pre-test aprueban } \\
11.1 \% \text { y en el post-test } 99.1 \%) \text {. }\end{array}$ \\
\hline $\begin{array}{l}\text { MORA, M. Y } \\
\text { CAMACHO, J. } \\
\text { (2019). }\end{array}$ & España & $\begin{array}{l}\text { Facilitar la adquisición de la gramática } \\
\text { básica del inglés al utilizar la } \\
\text { plataforma Classcraft y motivar al } \\
\text { alumnado con estrategias de } \\
\text { gamificación. }\end{array}$ & $\begin{array}{l}\text { Alumnos de } 6^{\circ} \text { educación primaria } \\
\text { que estudian inglés. No especifica el } \\
\text { número de participantes. }\end{array}$ & $\begin{array}{l}\text { Classcraft ofrece muchas } \\
\text { posibilidades educativas. Su uso } \\
\text { mejora las capacidades lingüísticas } \\
\text { del alumnado y el proceso de } \\
\text { enseñanza-aprendizaje de la lengua } \\
\text { inglesa en educación primaria. }\end{array}$ \\
\hline MORA, P. M. (2016). & Colombia & $\begin{array}{l}\text { Aplicación de una estrategia que } \\
\text { vincula el uso de la tecnología con } \\
\text { procesos de enseñanza-aprendizaje } \\
\text { (Kahoot!). }\end{array}$ & $\begin{array}{l}\text { Estudiantes universitarios que } \\
\text { cursan la asignatura "Didáctica del } \\
\text { español como lengua extranjera" e } \\
\text { "Intercultural Communication II" del } \\
\text { grado en Lengua Castellana, Inglés } \\
\text { y Francés en la Universidad La Salle } \\
\text { (Bogotá). No especifica el número } \\
\text { de participantes. }\end{array}$ & $\begin{array}{l}\text { La integración de tecnologías y de } \\
\text { la gamificación facilita el } \\
\text { aprendizaje. }\end{array}$ \\
\hline $\begin{array}{l}\text { OREJUDO, J. P. } \\
\text { (2019). }\end{array}$ & EEUU & $\begin{array}{l}\text { Explicación e implementación de cómo } \\
\text { gamificar tareas de lectura siguiendo } \\
\text { la teoría de Brown y Lee (2005). }\end{array}$ & $\begin{array}{l}\text { Dos grupos de estudiantes de ELE } \\
\text { de Texas A\&M University- } \\
\text { Commerce (grupo de control y } \\
\text { experimental, niveles B1-2). No } \\
\text { especifica el número de } \\
\text { participantes. }\end{array}$ & $\begin{array}{l}\text { La gamificación puede usarse en } \\
\text { clases de segundas lenguas para } \\
\text { crear rutinas positivas y completar } \\
\text { adecuadamente tareas de lectura. }\end{array}$ \\
\hline $\begin{array}{l}\text { PARDOEL, B, } \\
\text { PAPADIMA- } \\
\text { SOPHOCLEOUS, S. Y } \\
\text { ATHANASIOU, A. } \\
\text { (2018). }\end{array}$ & $\begin{array}{l}\text { Países } \\
\text { Bajos }\end{array}$ & $\begin{array}{l}\text { Examinar cómo la gamificación afecta } \\
\text { el aprendizaje de lenguas extranjeras } \\
\text { a alumnos de educación secundaria. }\end{array}$ & $\begin{array}{l}\text { Dos grupos de } 39 \text { alumnos de } \\
\text { educación secundaria ( } 13-14 \text { años) } \\
\text { que estudian alemán como lengua } \\
\text { extranjera en Países Bajos. }\end{array}$ & $\begin{array}{l}\text { Un curso de lengua extranjera } \\
\text { gamificado funciona más } \\
\text { positivamente con tiempos acotados } \\
\text { (máximo cuatro semanas). Se } \\
\text { requiere mayor andamiaje y ayuda } \\
\text { al estudiante en las primeras etapas } \\
\text { de implantación. }\end{array}$ \\
\hline $\begin{array}{l}\text { PÉREZ, L. M. } \\
\text { (2018). }\end{array}$ & España & $\begin{array}{l}\text { Involucrar a los alumnos en su } \\
\text { proceso de aprendizaje de ELE a la } \\
\text { par que adquieren las competencias y } \\
\text { habilidades digitales. }\end{array}$ & $\begin{array}{l}24 \text { alumnos universitarios que } \\
\text { estudian ELE ( nivel B1-2) y } \\
\text { diferentes nacionalidades. }\end{array}$ & $\begin{array}{l}\text { Classcraft es un videojuego que } \\
\text { favorece el desarrollo cognitivo y el } \\
\text { trabajo colaborativo. Instrumento } \\
\text { motivador que promueve un } \\
\text { ambiente relajado y reduce los } \\
\text { niveles de estrés y ansiedad ante el } \\
\text { aprendizaje de ELE. }\end{array}$ \\
\hline PERRY, B. (2015). & Canadá & $\begin{array}{l}\text { Implementación del prototipo Explorez. } \\
\text { Evaluación de la herramienta y } \\
\text { percepción del alumnado. }\end{array}$ & $\begin{array}{l}11 \text { estudiantes universitarios de } \\
\text { primer año que estudian francés } \\
\text { como lengua extranjera (18-19 } \\
\text { años). }\end{array}$ & $\begin{array}{l}\text { Mejora del aprendizaje y de la } \\
\text { motivación del alumnado. }\end{array}$ \\
\hline $\begin{array}{l}\text { RAWENDY, D., } \\
\text { YING, Y., ARIFIN, Y. } \\
\text { Y ROSALIN, K. } \\
\text { (2017). }\end{array}$ & Indonesia & $\begin{array}{l}\text { Comprobar si Game Chinese } \\
\text { Language Learning tiene resultados } \\
\text { efectivos en el aprendizaje de la } \\
\text { lengua. }\end{array}$ & $\begin{array}{l}132 \text { estudiantes de } 4^{\circ}, 5^{\circ} \text { y } 6^{\circ} \text { curso } \\
\text { de primaria de escuelas de Jakarta } \\
\text { occidental ( } 9 \text { - } 12 \text { años) que estudian } \\
\text { chino. }\end{array}$ & $\begin{array}{l}\text { El uso de Game Chinese Language } \\
\text { Learning motivó al alumnado y } \\
\text { mostró mejores resultados } \\
\text { académicos que el aprendizaje } \\
\text { tradicional. }\end{array}$ \\
\hline $\begin{array}{l}\text { SÁNCHEZ, M. B. } \\
\text { (2018). }\end{array}$ & Ecuador & $\begin{array}{l}\text { Desarrollar una estrategia de } \\
\text { gamificación para la adquisición de } \\
\text { vocabulario en inglés de nivel pre- } \\
\text { intermedio. }\end{array}$ & $\begin{array}{l}60 \text { estudiantes universitarios del } \\
\text { grado de Odontología de la UDLA } \\
\text { de Quito que cursan la asignatura } \\
\text { Inglés como lengua extranjera (nivel } \\
\text { B1). }\end{array}$ & $\begin{array}{l}\text { La implementación de la estrategia } \\
\text { de gamificación ha producido } \\
\text { cambios significativos en la forma } \\
\text { de adquirir la competencia léxica. }\end{array}$ \\
\hline $\begin{array}{l}\text { SARANGO, I. F. } \\
\text { (2018). }\end{array}$ & Ecuador & $\begin{array}{l}\text { Mejorar la atención, concentración, } \\
\text { convivencia, identidad y autonomía de } \\
\text { niños de } 3-5 \text { años al desarrollar } \\
\text { actividades gamificadas en el aula. }\end{array}$ & $\begin{array}{l}\text { Niños de } 3-5 \text { años de los CEIB de } \\
\text { Ecuador que estudian kichwa. No } \\
\text { especifica el número de } \\
\text { participantes. }\end{array}$ & $\begin{array}{l}\text { Adquisición de nuevas estrategias } \\
\text { de aprendizaje y refuerzo de lo } \\
\text { aprendido en clase. }\end{array}$ \\
\hline
\end{tabular}




\begin{tabular}{|c|c|c|c|c|}
\hline & & $\begin{array}{l}\text { Fomentar el interés por el aprendizaje } \\
\text { del idioma kichwa en niños de } 3-5 \\
\text { años mediante la implementación de } \\
\text { técnicas de gamificación en el proceso } \\
\text { educativo en los CEIB (Colegios de } \\
\text { Educación Intercultural Bilingües) para } \\
\text { evitar el posible riesgo de la pérdida } \\
\text { de dicho idioma. }\end{array}$ & & $\begin{array}{l}\text { Desarrollo de destrezas de } \\
\text { aprendizaje. }\end{array}$ \\
\hline $\begin{array}{l}\text { TEJEDOR, C. } \\
\text { (2016). }\end{array}$ & España & $\begin{array}{l}\text { Desarrollar una aplicación móvil para } \\
\text { mejorar la pronunciación en lengua } \\
\text { extranjera utilizando técnicas de } \\
\text { gamificación y social gaming. }\end{array}$ & $\begin{array}{l}\text { Estudiantes universitarios } \\
\text { norteamericanos que estudian ELE, } \\
\text { estudiantes españoles que estudian } \\
\text { Filología Inglesa y estudiantes } \\
\text { españoles del grado en Ingeniería } \\
\text { Informática con nivel B1 o B2 (todos } \\
\text { entre } 18 \text { - } 26 \text { años). No especifica el } \\
\text { número de participantes. }\end{array}$ & $\begin{array}{l}\text { Las TTS y ASR ayudan a la mejora } \\
\text { de pronunciación de lengua } \\
\text { extranjera tras una práctica } \\
\text { continuada. Los elementos de } \\
\text { gamificación contribuyeron a } \\
\text { incrementar la motivación y evitar el } \\
\text { abandono temprano. } \\
\text { La retroalimentación es necesaria } \\
\text { para poder mejorar la } \\
\text { pronunciación. }\end{array}$ \\
\hline VALLEJO, B (2019). & España & $\begin{array}{l}\text { Analizar el efecto de una experiencia } \\
\text { didáctica gamificada en un aula de } \\
\text { EFL de alumnos adultos (comprobar } \\
\text { su influencia en los aspectos afectivos } \\
\text { del aprendizaje de EFL, en el grado de } \\
\text { esfuerzo y participación en las } \\
\text { actividades académicas y en la } \\
\text { motivación del alumnado). }\end{array}$ & $\begin{array}{l}57 \text { estudiantes de Inglés con nivel } \\
\text { A2 (17-54 años) E.O.I. de } \\
\text { L'Hospitalet de Llobregat. }\end{array}$ & $\begin{array}{l}\text { Mayor participación. El entorno } \\
\text { distendido favorece las relaciones } \\
\text { sociales y disminuye el miedo a } \\
\text { cometer errores. Disminuye la } \\
\text { ansiedad. } \\
\text { El elemento de disfrute propicia una } \\
\text { asistencia más regular a clase pues } \\
\text { los alumnos están más motivados y } \\
\text { participan más activamente en su } \\
\text { aprendizaje. }\end{array}$ \\
\hline
\end{tabular}

\section{Discusión y Conclusión}

Dieciséis de los treinta estudios analizados tienen lugar en Europa, y catorce de ellos en España. América produce once investigaciones, donde Ecuador se posiciona en primer lugar con cuatro trabajos. Finalmente, tres estudios tienen lugar en Asia (Hong Kong, Indonesia y Filipinas). La mayoría de las publicaciones se centran en el aprendizaje de la lengua inglesa (quince estudios). Siete trabajos versan sobre el uso de la gamificación en la enseñanza de la lengua española, dos sobre el aprendizaje de francés, y uno sobre alemán, chino y kichwa. Tres trabajos tratan el aprendizaje de varias lenguas extranjeras y la aplicación de la gamificación (inglés, alemán, francés e italiano).

Los resultados mostrados en la tabla 1 constatan que el uso de la gamificación es todo un acierto en el aula de lenguas extranjeras. Las experiencias gamificadas analizadas muestran que los participantes se han implicado más en el aprendizaje de la lengua extranjera, con una actitud más positiva, colaborativa, participativa y responsable. El elemento del disfrute y el ambiente relajado ha propiciado unas sesiones más distendidas donde se ha reducido el estrés, la ansiedad y el miedo a cometer errores. La gamificación fomenta la interdependencia positiva y la interacción estimuladora del aprendizaje cooperativo, colaborativo y competitivo.

Los resultados académicos y el nivel de competencia lingüística (ya sea para mejorar la competencia léxica u oral, comprensión lectora, pronunciación, etc.) han mejorado significativamente tras implantar prácticas de gamificación. El uso de videojuegos y de información audiovisual en plataformas digitales con técnicas gamificadas favorece el desarrollo cognitivo, tiene múltiples posibilidades educativas y facilita el aprendizaje de lenguas extranjeras.

No obstante, el uso de la gamificación en el aula debe ser acotado. Su práctica no debe exceder las cuatro semanas para que el alumnado no caiga en la desidia y se precisa un mayor andamiaje y apoyo en las primeras etapas de implantación. Además, la retroalimentación constante es necesaria en todo su proceso. 
Tras analizar los resultados de las diferentes investigaciones analizadas, se pueden señalar las siguientes conclusiones: el uso de la gamificación en la enseñanza de lenguas extranjeras estimula la participación activa de los estudiantes en su propio desarrollo de aprendizaje de dicha lengua, además de motivar constantemente al alumnado y promueve la perseverancia. Por otro lado, ayuda a fomentar la comunicación y el trabajo cooperativo y colaborativo entre iguales y crea ambientes de confianza, disminuyendo así el estrés y la ansiedad que se puede experimentar en el aula. Finalmente, favorece la autoestima y mejora la tolerancia del error.

Para realizar una práctica gamificada con éxito debe haber un equilibrio entre actividad lúdica, destrezas personales, conocimientos previos, adquisición de nuevos contenidos y desafíos. El progreso así resulta más fácil y el tiempo pasa rápidamente. Con este tipo de experiencias, los estudiantes disfrutan de su aprendizaje y mejoran su proceso de aprendizaje de manera cualitativa y cuantitativa.

\section{Referencias}

Bárcena, E. y Sanfilippo, M. (2015). La píldora informativa audiovisual como estrategia de gamificación en los cursos en línea de segundas lenguas. Círculo De Lingüística Aplicada a La Comunicación, 63 , 122-151.

Barros, M. (2016). La gamificación en el aula de lengua extranjera. En: Secretaria General Técnica. Ministerio de Educación, Cultura y Deporte (ed), El español como lengua extranjera en Portugal II: retos de la enseñanza de lenguas cercanas (pp.14-25). Madrid: Centro de Publicaciones Ministerio de Educación, Cultura y Deporte.

Bartle, R. (19.07.2019). Hearts, clubs, diamonds, spades: Players who suit MUDs. [http://mud.co.uk/richard/hcds.htm]

Battle, J. (2016). Gamificación para el desarrollo de la comunicación intercultural en el aula de español como lengua extranjera. En R. Roig-Vila (Ed.), Tecnología, innovación e investigación en los procesos de enseñanza-aprendizaje (pp.114-120). Barcelona: Octaedro .

Batlle, J., González, MV. y Pujolà, JT. (2018). La narrativa como elemento cohesionador de tareas gamificadas para la enseñanza de lenguas extranjeras. Rivista dell'Istituto di Storia dell'Europa Mediterranea, 2/II, 121-160

Batlle, J. y Suárez, MM. (2019). Secuencias didácticas gamificadas por docentes de LE en formación continua: puntos, insignias y tablas de clasificación. E-Aesla 5, 43-51.

Cabarique, LI. (2018). Influencia de los juegos de programas tipo talk show trabajados bajo elementos de la gamificación en el desempeño de la producción oral en inglés en adultos de la fundación ColombiaCrece. Trabajo Fin de Grado. Bogotá: Pontificia Universidad Javeriana.

Carrión-Salinas, GA. (2017). Gamificación en educación primaria. Un estudio piloto desde la perspectiva de sus protagonistas. Trabajo Fin de Máster. Loja: Universidad Técnica Particular de Loja.

Cordero, D. y Núñez, VM. (2018). El uso de técnicas de gamificación para estimular las competencias lingüísticas de estudiantes en un curso de ILE. Revista de Lenguas Modernas, 28, 269-291.
Cruaud, C. (2017). The Playful Frame: Design and Use of a Gamified Application for Foreign Language Learning. Tesis doctoral (PhD) Universitetet I Oslo.

Csikszentmihalyi, M. (1990). Flow. New York: Harper and Row.

Dalmases, A. (2017). Uso de la gamificación en la enseñanza de ELE. E-eleando: Ele en Red. Serie de monografías y materiales para la enseñanza de ELE, 1(4), 1-74.

Deterding, S., Dixon, D., Khaled, R., \& Nacke, L. (2011). From game design elements to gamefulness: defining 'gamification'. Proceedings of the 15th International Academic MindTrek Conference: Envisioning Future Media Environments (MindTrek 11). Tampere (Finland) - September 28-30, 2011. ACM, New York, (NY), 9-15.

Díaz, JJ. y Cerda GM. (2018). La gamificación como estrategia correctiva para la interferencia sintáctico-morfológica del español en la producción escrita del idioma inglés de los estudiantes de nivel pre-intermedio del programa de suficiencia en la universidad de las fuerzas armadas ESPE extensión Latacunga durante el período octubre 2016-marzo 2017. Trabajo Fin de Máster. Quito: UCE.

Foncubierta, JM. (2014). Gamificación y aprendizaje de segundas lenguas, Madrid: Edinumen.

Galeano, JD. y Rodríguez LE. (2016). El uso de las técnicas de gamificación en la adquisición de vocabulario y el dominio de los tiempos verbales en inglés. Trabajo Fin de Grado. Bogotá: Universidad Pedagógica Nacional.

García, F. (2017). El uso de la gamificación para la mejora de la escritura y aprendizaje de lengua extranjera en educación primaria. Tesis doctoral (PhD). Madrid: Universidad Camilo José Cela.

Gargallo, P. (2017). Una experiencia de gamificación con tablets para potenciar el inglés en aula de infantil. Trabajo Fin de Grado. Castellón: Universitat Jaume I.

González, D. (2017). La gamificación como elemento motivador en la enseñanza de una segunda lengua en educación primaria. Trabajo Fin de Grado. Burgos: Universidad de Burgos.

Hammer, J., \& Lee, J. J. (2011). Gamification in Education: What, How, Why Bother? Academic Exchange Quarterly, 15(2), 1-5. 
Huizinga, J. (1949). Homo Ludens. A study of the play-element in culture. London: Routledge \& Kegan Paul.

Ibáñez, A. (2015). La gamificación para (auto)evaluar las competencias léxico-gramaticales en el aula de inglés como segunda lengua en el contexto de la enseñanza a distancia: un estudio de caso. Verbeia, 0, 75-99.

Jiménez, D. (2015). La gamificación en la enseñanza de Español como Lengua Extranjera. Análisis y propuestas de aplicaciones con estrategias ludificadas. Tesis Doctoral (PhD). Universidad de Sevilla.

Lam, YW., Hew, KF., \& Chiu, KF. (2018). Improving Argumentative Writing: Effects of a Blended Learning Approach and Gamification. Language Learning \& Technology, 22(1), 97-118.

Martín-Macho, A. y Faya, F. (2016). El juego en el aula de lengua inglesa para consolidar contenidos: experiencia con futuros docentes de Educación infantil. En A. Díez, V. Brotons, D. Escandell y J. Rovira (Eds). Aprendizajes plurilingües y literarios. Nuevos enfoques didácticos (pp. 873-878). Sant Vicent del Raspeig: Universitat D'Alacant.

Mongrovejo, AB., Mamani, G. y Tipo, ML. (2019). Juego y simulación de programas concurso de televisión como técnica didáctica para mejorar el aprendizaje del vocabulario inglés en estudiantes de habla hispana. Información tecnológica, 30(1), 225236.

Mora, F. (2013). Neuroeducación. Solo se puede aprender aquello que se ama. Madrid: Alianza Editorial.

Mora, M. y Camacho, J. (2019). Classcraft: inglés y juego de roles en el aula de educación primaria. Apertura, 11(1), 56-73.

Mora, PM. (2016). Percepciones de los estudiantes sobre la articulación entre la tecnología con el juego y el trabajo colaborativo para el aprendizaje de lenguas. Trabajo Fin de Grado. Bogota: Universidad de La Salle.

Orejudo, JP. (2019). Gamificar tareas de lectura en una segunda lengua: un estudio preliminar. Revista de Estudios y Experiencias en Educación, 18(36), 95-103.

Pardoel, B, Papadima-Sophocleous, S., \& Athanasiou, A. (2018). How MISSION BERLIN Gamified my FL/L2 German Class. A Six Week Journey. En P. Taalas, J. Jalkanen, L. Bradley y S. Thouêsny (Eds). Future-proof CALL: Language Learning as Exploration and Encounters- short papers from EUROCALL 2018. (255-260).

Pérez, LM. (2018). ¿Estoy en clase de ELE o en un videojuego? En REDINE (ed). EDUNOVATIC 2018. 3RD Virtual International Conference on Education, Innovation and ICT. Eindhoven: Adaya Press, 50-54.

Perry, B. (2015). Gamifying French Language Learning: A Case Study Examining a Quest-based Augmented Reality Mobile Learning Tool. Procedia -Social and Behavioral Sciences, 174, 2308-2315.

Rawendy, D., Ying, Y., Arifin, Y., \& Rosalin, K. (2017). Design and Development Game Chinese Language Learning with Gamification and Using Mnemonic Method. Procedia Computer Science, 116, 6167
Sánchez, MB. (2018). Gamificación: una estrategia para la adquisición de vocabulario del idioma inglés nivel pre-intermedi. Trabajo Fin de Máster. Ambato: Pontificia Universidad Católica del Ecuador.

Sarango, IF. (2018). Innovación educativa: gamificación del proceso educativo a niños de 4-5 años mediante un aplicativo web para desarrollar el aprendizaje del idioma kichwa. Caso CDTI-ITSCO. Trabajo Fin de Grado. Quito: Tecnológico Superior Cordillera.

Tejedor, C. (2016). Tip Top Talk! Aplicación móvil para la mejora de la pronunciación multilingüe mediante pares mínimos y gamificación. Trabajo Fin de Máster. Valladolid: Universidad de Valladolid.

Vallejo, B. (2019). Implicaciones de una propuesta gamificada en la dimensión afectiva del alumnado adulto de EFL. E-Aesla, 5, 85-96.

Werbach, K. (15.07.2019). Gamification, University of Pennsylvania. [https:/les.coursera.org/learn/gamification]

Werbach, K., \& Hunter, D. (2012). For the Win: How Game Thinking Can Revolutionize Your Business. PA: Wharton Digital Press.

Zichermann, G., \& Cunningham, C. (2011). Gamification by Design: Implementing Game Mechanics in Web and Mobile Apps. Cambridge, MA: O'Reilly Media. 